\title{
Severe Acute Respiratory Syndrome (SARS)
}

\author{
G. Bhaskar, Rakesh Lodha and S.K. Kabra \\ Department of Pediatrics, All India Institute of Medical Sciences, Ansari Nagar, New Delhi 110029
}

\begin{abstract}
Several cases of life threatening respiratory disease with no identifiable cause were reported from Guangdong Province, China; these were soon followed by reports from many other countries. The disease was named as severe acute respiratory syndrome (SARS). A novel coronavirus, isolated from the respiratory secretions of patients, has been implicated in the causation of SARS. The modes of transmission include droplet spread, close contact, and Fomites; shedding of virus from respiratory tract is the primary mode of transmission. SARS clinically presents with high-grade fever, chills and rigors, myalgia, headache, cough with or without sputum production, dyspnea, and dizziness. Chest radiographs reveal unilateral or bilateral, predominantly peripheral, areas of consolidation progressing with in a short time of bilateral patchy consolidation. Preliminary reports suggest a milder illness in young children. The case definition of probable SARS cases, laboratory investigations and precautions for prevention of spread are discussed. [Indian J Pediatr 2003; 70 (5) : 401-405]
\end{abstract}

Key words : SARS; Diagnosis; Children

In late 2002, several cases of life threatening respiratory disease with no identifiable cause were reported from Guangdong Province, China. These were followed by reports from Hong Kong, Singapore, Vietnam, United States, Canada and many other countries. The syndrome was designated "Severe acute respiratory syndrome (SARS)" in late February 2003 and global efforts to understand the cause of illness and prevent its spread were instituted in March 2003.

The WHO issued a global alert about SARS and Centers of Disease Control and Prevention (CDC, USA) activated its emergency operations, to support the response of WHO to this global threat. As of May 3, 2003 there were 6234 probable cases of SARS reported worldwide and 435 deaths related to the illness. ${ }^{1}$ The maximum numbers have been reported from China and Hong Kong.

\section{Etiology}

A novel coronavirus has been isolated from the respiratory secretions of a patient with SARS and subsequently this virus or a serologic response to this virus has been demonstrated in other patients. This points to a possible etiologic association between this novel coronavirus and SARS. ${ }^{24}$

Coronaviruses, RNA viruses of which 3 groups are known, are associated with a variety of diseases in humans and domestic animals including gastroenteritis and upper and lower respiratory tract disease. However, the known human coronavirus is associated with mild disease (the common cold). The ability of coronavirus to cause severe disease in animals raises the possibility that

Reprint requests : Dr. S.K. Kabra, Deptt. of Pediatrics, AIIMS, New Delhi-110029. E-mail : skkabra@hotmail.com coronavirus could also cause a more severe disease in humans.

The coronavirus implicated in SARS (SARS associated coronavirus) has got only $50-60 \%$ homology in nucleotide component to the known human coronaviruses. ${ }^{2}$ As expected with a point source epidemic, several nucleotide sequences of the viruses isolated from patients from different regions are identical. ${ }^{2,3}$

The apparent lack of antibody in all serum specimens except for those from patients with SARS suggests that this virus has not circulated previously. Presumably, this virus originated in animals and mutated or recombined in a fashion that permitted it to infect, cause disease and pass on from person to person. ${ }^{2}$

The name suggested for this new coronavirus is urbani SARS associated coronavirus in memory of Dr Carlo Urbani, the 40 year old WHO physician and infectious disease specialist whose work defined SARS, who died on March 29 of SARS. ${ }^{2}$

Metapneumovirus was isolated in 5 patients in one study, ${ }^{5}$ but further studies did not reveal the same.

\section{MODE OF TRANSMISSION}

The infection appears to be highly but not uniformly contägious. Secondary attack rates are about $50 \% .{ }^{5.7}$ In one study on children, it was suggested that infected children did not spread the infection to other children in their class in school. ${ }^{8}$

The following may be modes of transmission: Droplet spread, Close contact, Fomites; shedding of virus in feces may be an additional source of spread. Viral RNA concentrations in sputum were noticed to be high, suggesting that the shedding of virus from respiratory 


\section{G. Bhaskar et al}

TABle 1. Clinical and Laboratory Features in Patients with SARS.

\begin{tabular}{|c|c|c|c|c|}
\hline Variable & $\begin{array}{l}\text { Poutanen } e t a l^{5} \\
\mathrm{~N}=10\end{array}$ & $\begin{array}{l}\text { Lee et } a l^{6} \\
\mathrm{~N}=138\end{array}$ & $\begin{array}{l}\text { Tsang et } a l^{7} \\
\mathrm{~N}=10\end{array}$ & $\begin{array}{l}\text { Peiris et al } \\
\mathrm{N}=50\end{array}$ \\
\hline \multicolumn{5}{|l|}{ Symptoms } \\
\hline Fever & $100 \%$ & $100 \%$ & $100 \%$ & $100 \%$ \\
\hline Chills/rigors & - & $73.2 \%$ & $90 \%$ & $74 \%$ \\
\hline Myalgia & $70 \%$ & $60.9 \%$ & $50 \%$ & $54 \%$ \\
\hline Cough & $100 \%$ & $57.3 \%$ & $80 \%$ & $62 \%$ \\
\hline Headache & $30 \%$ & $55.8 \%$ & $70.1 \%$ & \\
\hline Sputum production & - & $29 \%$ & $10 \%$ & \\
\hline Sore throat & $30 \%$ & $23.2 \%$ & 0 & $20 \%$ \\
\hline Coryza & - & $22.5 \%$ & $10 \%$ & $24 \%$ \\
\hline \multicolumn{5}{|l|}{ Investigations } \\
\hline Infiltration on chest radiogram & $100 \%$ & $96.3 \%$ & $100 \%$ & $100 \%$ \\
\hline Oxygen saturations on room air $(<95 \%)$ & $70 \%$ & - & $60 \%$ & - \\
\hline \multicolumn{5}{|l|}{ Lencopenia } \\
\hline$\left(<3.5 \times 10^{4} / \mathrm{L}\right)$ & $22 \%$ & $33.9 \%$ & $10 \%$ & $26 \%$ \\
\hline \multicolumn{5}{|l|}{ Lymphopennia } \\
\hline$\left(<1.5 \times 10^{9} / \mathrm{L}\right)$ & $89 \%$ & $69.6 \%$ & $60 \%$ & $68 \%$ \\
\hline \multicolumn{5}{|l|}{ Thrombocytopenia } \\
\hline$\left(<1.5 \times 10^{9} / \mathrm{L}\right)$ & $33 \%$ & $44.8 \%$ & $20 \%$ & $40 \%$ \\
\hline LDH (above upper limit of normal) & $80 \%$ & $71 \%$ & - & - \\
\hline AST (above upper limit of normal) & $78 \%$ & - & $60 \%$ & - \\
\hline $\operatorname{ALT}(>45 \mathrm{IU})$ & $56 \%$ & $23.4 \%$ & $60 \%$ & $34 \%$ \\
\hline
\end{tabular}

tract is the primary mode of transmission ${ }^{2}$. SARS also appears to spread by direct contact with respiratory secretions, which makes touching contaminated objects a potential concern. Coronavirus is light sensitive but can survive in body secretions for few hours.

\section{CLINICAL FEATURES}

The incubation period for SARS has been observed to be 2-11 days, with a median of 6 days. ${ }^{5-7}$ The clinical and laboratory features reported in various series are given in Table 1 . SARS clinically presents with high-grade fever, chills and rigors, myalgia, headache, cough with or without sputum production, dyspnea, and dizziness. It is less commonly associated with sore throat, coryza, nausea and vomiting and diarrhea. Rash, purpura and lymphadenopathy are not features of SARS. ${ }^{5-7}$

Physical examination may reveal increased respiratory rate, dull percussion note in chest and inspiratory crackles in lower chest.

On investigation, some patients were found to have low arterial oxygen saturation. Chest radiographs have revealed unilateral or bilateral, predominantly peripheral, areas of consolidation progressing within a short time to bilateral patchy consolidation. ${ }^{6}$ Characteristic chest $\mathrm{CT}$ findings observed include bilateral air space ground glass consolidation mimicking that seen in bronchiolitis obliterans with organizing pneumonia. ${ }^{5,6}$

Laboratory data has revealed leucopenia, lymphopenia and thrombocytopenia, deranged APTT, abnormal Ddimer assay. ${ }^{57}$ Serum AST, ALT, LDH and CK levels were found to be raised. Electrolyte abnormalities in the form of hypenatremia and hypokalemia have been observed in a few patients. ${ }^{5-7}$

A recent study on 75 adults with SARS has observed that the fever and pneumonia initially responded to treatment. ${ }^{9}$ However, patients developed recurrent fever $(85.3 \%$ ) on day $8.9 \pm 3.1$ (range 4 to 18 ), watery diarrhea $(73.3 \%$ ) on day $7.5 \pm 2.3$ (range 3 to 15 ), radiological deterioration $(80 \%$ ) on day $7.4 \pm 2.2$ (range 3 to 13 ) and respiratory deterioration ( $45.3 \%$ ) on day $8.6 \pm 3$ (range 5 to 19). In $45.3 \%$ of patients, marked improvement of initial pulmonary lesions was closely associated with appearance of new radiological lesions at other sites. Twenty percent progressed to acute respiratory distress syndrome (ARDS) during the third week. Quantitative RT-PCR of nasopharyngeal aspirates in 14 patients ( 4 had ARDS and 10 without ARDS) consistently demonstrated a peak viral load at day 10 and a decrease to admission level at day 15 . This suggests that deterioration during the second week was not related to uncontrolled viral replication but may rather be related to immunopathological damage."

Data on children is limited. Hon et al have described the clinical features in 10 children from Hong Kong. ${ }^{8}$ Two distinct patterns of clinical presentation among the children were observed. Teenaged patients presented with symptoms of malaise, myalgia, chill, and rigor similar to those of adults, whereas the younger children presented mainly with cough and runny nose, and none had chills, rigors, or myalgia. The clinical course was much milder and shorter among younger patients, and radiological changes were milder and generally resolved more quickly than in the teenagers. All pediatric patients 


\section{Severe Acute Respiratory Syndrome (SARS)}

had clinically important lymphopenia, but it was more severe among the teenage children.

\section{Postmortem Findings ${ }^{6}$}

Postmortem examination in a few cases has shown gross consolidation of lungs. The early phase and organizing phase of diffuse alveolar damage were seen in different parts of lung. The early phase was characterized by pulmonary edema and hyaline membrane formation suggestive of early phase of ARDS. Cellular fibromyxoid organizing exudates in air spaces indicated the ongoing phase of alveolar damage. It was also noticed that there was a scanty lymphocytic inflammatory infiltrate in the interstitium; vacuolated and multinucleated pneumocytes were also identified, no viral inclusions were detected. There was no evidence of involvement of other organs.

\section{Diagnosis and Evaluation ${ }^{10,11}$}

Diagnosis is mainly based on clinical features and exclusion of other known causes of acute lower respiratory tract infection (community acquired pneumonia, atypical pneumonia and other viral pneumonia).

New case definitions that include laboratory test criteria are under final review and productions of test reagents for widespread distribution are well underway.

The symptoms and laboratory finding in SARS are non specific and constellation of various clinical and biochemical parameters should alert the medical pracatitioners to the possibility of SARS.

The case definition of SARS is as follows ${ }^{10,11}$ :

\section{Suspect Case}

Respiratory illness of unknown etiology with onset of since February 1, 2003, and the following criteria:

1. Measured temperature greater than $100.4^{\circ} \mathrm{F}\left(>38^{\circ} \mathrm{C}\right)$, and

2. One or more clinical findings of respiratory illness (e.g. shortness of breath, difficulty breathing or hypoxia), and

3. Travel within 10 days of onset of symptoms to an area with documented or suspected community transmission of SARS (excludes areas with secondary cases limited to health case workers or direct household contacts).

\section{OR}

4. Close contact within 10 days of onset of symptoms with a person known to be a suspect SARS.

\section{Probable Case}

A suspect case with one of the following:

1. Presence of radiographic evidence of pneumonia or respiratory distress syndrome.

2. Presence of autopsy finding consistent with respiratory distress syndrome without an identifiable cause.
Travel : includes transit in an airport in an area with documented or suspected community transmission of SARS. Areas with documented or suspected community transmission of SARS: peoples Republic of China (i.e. mainland China and Hong Kong special administrative region); Hanoi, Vietnam; Singapore; Toronto, Canada.

Close Contact : is defined as having cared for, having lived with, or having direct contact with respiratory secretions and or body fluids of a patient known to be a suspect SARS case.

\section{Laboratory Methods helpful in Diagnosis}

Investigations that may help in making a diagnosis of SARS include $X$ ray film of chest, cultures of sputum, nasopharyngeal aspirates, throat swabs, blood and serological tests to exclude known causes of various pneumonias. Specific test ${ }^{2}$ include viral cultures (highest yield seen with sputum), RT PCR assays and serological tests such as ELISA and Indirect fluorescent antibody test (positive after 14 days the onset of illness). However, serological tests at the moment may not be very specific because of cross reactivity with other coronaviruses. In India, the diagnostic tests are available at National Institute of Communicable Diseases (NICD), Delhi and National Institute of Virology (NIV), Pune. Because of lack of reliable and easy to perform tests, the current case definition is based on clinical and radiological criteria. One should try to exclude common causes of atypical pneumonia.

\section{Treatment $^{12}$}

Numerous antibiotics (clarithromycin, third generation cephalosporins, levofloxacin) and anti-influenza therapies have been tried with no clear effect. Physicians in SARS affected areas have used ribavarin with or without use of steroids in an increasing number of patients. ${ }^{6,8,9} \mathrm{But}$, in the absence of clinical indicators, its effectiveness has not been proven. Supportive care is particularly important.

\section{Prognosis}

Overall case fatality is about $4 \%$. Lee $\mathrm{et}^{6} \mathrm{l}^{6}$ observed that in a multivariate analysis, the independent predictors of an adverse outcome were advanced age (odds ratio per decade of life, 1.80 ; 95 percent confidence interval, 1.16 to 2.81; $P=0.009$ ), a high peak lactate dehydrogenase level (odds ratio per $100 \mathrm{U}$ per liter, 2.09; 95 percent confidence interval, 1.28 to $3.42 ; \mathrm{P}=0.003$ ), and an absolute neutrophil count that exceeded the upper limit of the normal range on presentation (odds ratio, 1.60; 95 percent confidence interval, 1.03 to $2.50 ; \mathrm{P}=0.04){ }^{6}$

According to this study $32.2 \%$ required ICU admission and $13.8 \%$ required mechanical ventilatory support. Mortality rate was nearly $5 \%$.

One report on ten children observed that four teenagers required oxygen therapy and two needed assisted ventilation; none died. ${ }^{8}$ None of the younger 


\section{G. Bhaskar et al}

children required oxygen supplementation. This suggests that the disease is milder in children.

\section{Discharge Criteria ${ }^{13}$}

The WHO has recommended that the following criteria be considered prior to making a decision regarding discharge from hospital regarding a convalescent case:

\section{Clinical symptoms/findings}

- Afebrile for 48 hours

- Resolving cough

\section{Laboratory tests: if previously abnormal}

- White cell count returning to normal

- Platelet count returning to normal

- Creatine phosphokinase returning to normal

- Liver function tests returning to normal

- Plasma sodium returning to normal

- C reactive protein returning to normal

\section{Radiological findings:}

- Improving chest $X$-ray changes

Prevention ${ }^{14,} 15$

For all contact with suspect SARS patients, careful hand hygiene is urged, including hand washing with soap and water; if hand are not visibly soiled, alcohol-based handrubs may be used as an alternative to hand washing.

For the inpatients setting: If a suspect SARS patient is admitted to the hospital, infection control personnel should be notified immediately. Infection control measures for inpatients should include:

1. Standard precaution (e.g. hand hygiene); in addition to routine standard precaution, health-care personnel should wear eye protection for all patient contact.

2. Contact precautions (e.g. use of gown and gloves for contact with the patients or their environment).

3. Airborne precautions (e.g. an isolation room with negative pressure relative to the surrounding area and use of an N-95 filtering disposable respirator for persons entering the room).

If airborne precautions cannot be fully implemented, patients should be placed in a private room, and all persons entering the room should wear $\mathrm{N}-95$ respirators. If $\mathrm{N}-95$ respirators are not available for health-care personnel, surgical masks should be worn. Regardless of the availability of facilities for airborne precautions, standard and contact precautions should be implemented for all suspected SARS patients.

For the outpatient setting: If possible, suspect SARS patients on arrival to the outpatient or ambulatory setting e.g. clinic or Emergency Department (ED), should be evaluated in a separate assessment area to determine if they meet the case definition for suspected SARS and require isolation. A surgical mask should be placed on the patients, if possible.

All health-care personnel should wear $\mathrm{N}-95$ respirators
(95\% filter efficiency) while taking care of patients with suspected SARS. Precaution should be used when evaluating or transporting (e.g. emergency medical technicians), or in any ambulatory health-care setting (e.g. Emergency Department or clinic personnel). If N-95 respirators are not available, personnel should wear surgical masks.

For home or residential setting : Placing a surgical mask on suspect SARS patient during contact with others at home is recommended. If the patient is unable to wear a surgical mask, it may be prudent for household members to wear surgical masks when in close contact with the patient, should be reminded of the need for careful hand hygiene including hand washing with soap and water; if hands are not visibly soiled, alcohol-based handrubs may be used as an alternative to hand washing.

\section{REFERENCES}

1. World Health Organization. Cummulative number of reported probable cases of Severe Acute Respiratory Syndrome (SARS). (Accessed May 5, 2003 at http:// www.who.int/csr/sarscountry/2003_05_03/en/).

2. Ksiazek TG, Erdman D, Goldsmith CS, Zaki SR, Peret T, Emery $\mathrm{S}$ et al. A novel coronavirus associated with Severe Acute Respiratory Syndrome. N Engl J Med 2003; 348: 19471958.

3. Drosten C, Günther S, Preiser W, van der Werf S, Brodt H, Becker $S e t$ al. Identification of a novel coronavirus in patients with Severe Acute Respiratory Syndrome. Article published at www.nejm.org on April 10, 2003.

4. Peiris JSM, Lai ST, Poon LLM, Guan Y, Yam LYC, Lim W et al. Coronavirus as a possible cause of severe acute respiratory syndrome. Lancet 2003; 361: 1319-1325.

5. Poutanen SM, Low DE, Henry B, Finkelstein S, Rose D, Green $K$ et al. Identification of Severe Acute Respiratory Syndrome in Canada. Article published at www.nejm.org on March 31, 2003.

6. Lee N, Hui D, Wu A, Chan P, Cameron P, Joynt GM et al. A Major Outbreak of Severe Acute Respiratory Syndrome in Hong Kong. Article published at www.nejm.org on April 7, 2003 .

7. Tsang KW, Ho PL, Ooi GC, Yee WK, Wang T, Chan-Yeung M et al. A Cluster of Cases of Severe Acute Respiratory Syndrome in Hong Kong. Article published at www.nejm.org on March $31,2003$.

8. Hon KLE, Leung CW, Cheng WTF, Chan PKS, Chu WCW, Kwan YW et al. Clinical presentations and outcome of severe acute respiratory syndrome in children. Lancet. Published online April 29, 2003 (Accessed May 1, 2003 at http:// image.thelancet.com/extras/03let4127web.pdf).

9. Peiris JSM, Chu CM, Cheng VCC, Chan KS, Hung IFN, Poon LLM et al. Prospective study of the clinical progression and viral load of SARS associated coronavirus pneumonia in a community outbreak. Lancet (Accessed May 1, 2003 at http:// www.who.int/csr/sars/prospectivestudy/en/index.html).

10. Centers for Disease Control and Prevention. Severe acute respiratory syndrome: updated interim care definition (Accessed April 29, 2003 at http://www.cdc.gov/ncidod/ sars/casedefinition.htm).

11. World Health Organization. Case Definitions for Surveillance of Severe Acute Respiratory Syndrome (SARS). (Accessed May 1, 2003 at http://www.who.int/csr/sars/casedefinition/ en/)

Indian Journal of Pediatrics, Volume 70-May, 2003 


\section{Severe Acute Respiratory Syndrome (SARS)}

12. World Health Organization. Management of Severe Acute Respiratory Syndrome (SARS). (Accessed May 1, 2003 at http:/ /www.who.int/csr/sars/management/en/).

13. World Health Organization. WHO hospital discharge and follow-up policy for patients diagnosed with Severe Acute Respiratory Syndrome (SARS). (Accessed May 1, 2003 at http:/ /www.who.int/csr/sars/discharge/en/).

14. World Health Organization. Hospital Infection Control
Guidance for Severe Acute Respiratory Syndrome (SARS). (Accessed May 1, 2003 at http://www.who.int/csr/sars/ infectioncontrol/en/).

15. Centers for Disease Control and Prevention. Updated interim domestic infection control guidance in the health care and community setting for patients with suspected SARS (Accessed April 29, 2003, at http://www.cdc.gov/ncidod/ sars/infectioncontrol.htm). 